\title{
TITLE:
}

\section{Crystallization of polyethylene under high pressure}

$\operatorname{AUTHOR}(S)$ :

Osugi, Jiro; Hara, Kimihiko; Hirai, Nishio; Hikasa, Junichi

\section{CITATION:}

Osugi, Jiro ...[et al]. Crystallization of polyethylene under high pressure. The Review of Physical Chemistry of Japan 1965, 34(2): 59-64

\section{ISSUE DATE:}

1965-06-20

URL:

http://hdl.handle.net/2433/46850

RIGHT: 
The Review of Physical Chemistry of Japan, Vol. 34, No. 2, 1964

\title{
CRYSTALLIZATION OF POLYETHYLENE UNDER HIGH PRESSURE
}

\author{
By Jiro Osugi, Kiminiko Hara, Nishio Hirai* and Junichi Hikasa*
}

The crystallization and the annealing process of polyethylene under high pressure have been studied. The thickness of single crystal lamellae can be represented well by the equation of Lauritzen-Hoffman up to $2000 \mathrm{~atm}$ if one uses the melting temperature predicted by the Clapeyron equation. The surface free energy of the end surface of single crystal has been estimated, and been found to decrease with increasing pressure.

\section{Introduction}

Till, Keller and Fischer found independentlyli that polyethylene crystallizes isothermally from the dilute solution as single crystal lamellae. After this discovery many polymers were crystallized in the form of single crystal, and now it is believed that any crystallizable polymers can bê single crystals if proper solvents are chosen. The single crystal oi polyethylene is a lamella of lozenge shape with nearly the same basic thickness which is about $100 \mathrm{~A}$. It has been shown that the uniform thickness of plate-like crystals is associated with the fold configuration of the chain molecules which are perpendicular to the plane of the chain folds. The thickness of the lamella increases with the temperature of crystallization or annealing.

The experiment of the polymer crystallization under high pressure was done by Matsuoka at first.2) He found that the linear polyethylene crystallized under high pressure has a density approaching the perfect crystal density calculated from the crystal lattice constants obtained from $x$-ray analysis and its melting point is close to the limiting value estimated for the perfect crystals. Recently Geil and his coworkers3) found that the thickness of the crystal lamella of linear polyethylene crystallized from melt under pressure of about $5000 \mathrm{~atm}$ becomes extremely large and sometimes it amounts to the length of the polymer molecule itself, that is, about several thousands angstrom.

The present woik is carried out to study the mechanism of the process of the increase in the lamellar thickness under elevated pressure with rising temperature of crystallization.

\section{Experimentals}

(1) Preparation of samples

The samples used in this investigation were a linear polyethylene, Marlex $50.0 .2 \mathrm{~g}$ of Marlex

(Received, January 15, 1965)

1) P. H. Till, Jr., Polymer Sci., 24, 301 (1957)

A. Keller, Phil. Mag., 2, 1171 (1957)

E. W. Fischer, Z. Naturforsch. 12a, 753 (1957)

2) S. Matsuoka, J. Polymer Sci., 42, 511 (1960)

3) P. H. Geil, F. R. Anderson, B. Wunderlich and T. Arakawa, J. Polymer Sci., Part A 2, 3702 (1964)

* Department of Chemistry, Faculty of Science, Okayama University, Okayama, Japan 
50 was dissolved in $200 \mathrm{cc}$ of xylene at $120^{\circ} \mathrm{C}$. This solution was gradually added into xylene, whose volume was about 5 times as much as that of the solution, when the temperature was kept constant within $1^{\circ} \mathrm{C}$ above a given crystallization temperature. The suspension was aged isothermally for a few hours before filtration. After filtration and drying the thin film of about $0.05 \mathrm{~mm}$ thick was taken off from the filter paper. The single crystals were piled up in the film orienting their lamellar surface parallel to the film surface. The thin film was cut into small pieces of ribbon with a razor.

(2) Heat treatment under high pressure

The high pressure apparatus used is the piston cylinder type vessel with silicon oil as pressure transmitting medium, which produces the hydrostatic pressure (Fig. 1). The pressure and temperature were kept constant for $3 \mathrm{hrs}$ to accomplish the thickening process. Then the temperature was reduced to about $50^{\circ} \mathrm{C}$ at a rate of about $4^{\circ} \mathrm{C} / \mathrm{min}$ at which time the pressure was released and the sample removed. The small ribbons of the sample were charged in parallel in a sample holder, and submitted to the small angle $x$-ray measurement at room temperature. In order to obtain a sharp diffraction pattern as shown in Fig. 2, the $x$-ray beam should be directed parallel to the surface of

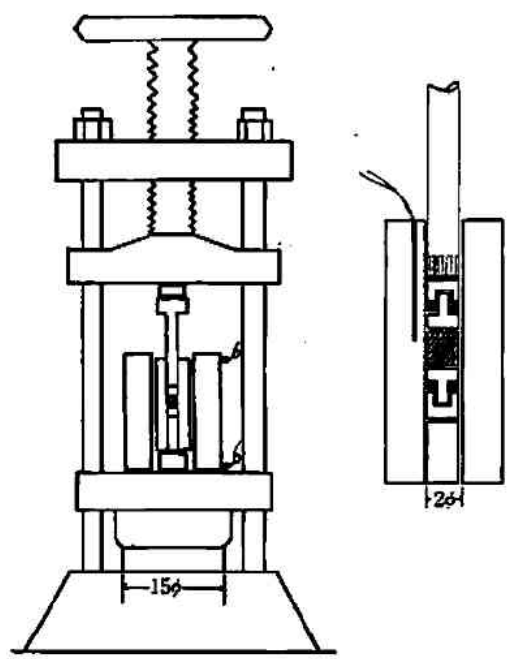

Fig. 1 The hydraulic press and piston cylinder type high pressure apparatus

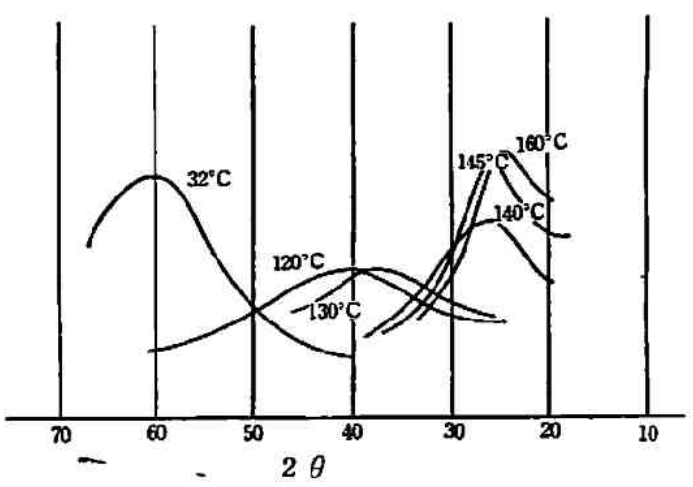

Fig. 2 Diffraction intensity curve of low angle x-ray for single crystal film of Marlex-50 annealed at various temperatures under $1,020 \mathrm{~atm}$ for $3 \mathrm{hrs}$

the film in which the single crystals were piled up with their flat surface parellel to the film surface.

(3) Crystallization from melt under high pressure

Pellets of Marlex 50 were enclosed in the above vessel under the chosen pressure and heated up to about $250^{\circ} \mathrm{C}$ to melt the polymer. Then it is reduced gradually down to $180^{\circ} \mathrm{C}$ and kept at the temperature and pressure for 3 hrs to accomplish the crystallization. After removal of the samples from the crystallization vessel, they were immersed in liquid nitrogen for a sufficient period of time to cool them and were then fractured by a hammer. The fractured surfaces were replicated by a direct platinum carbon technique, and the replicas were examined by means of an electron microscope. 


\section{Results}

(1) Heat treatment under high pressure

The thickness of the single crystals of polyetaylene annealed at atmospheric pressure increased with the rise of annealing temperature as shown in Fig. 3. A single crystal heated for $1.5 \mathrm{hr}$ at $120^{\circ} \mathrm{C}$ at atmospheric pressure is shown in Photo. 1. Comparison between the area of the annealed crystal

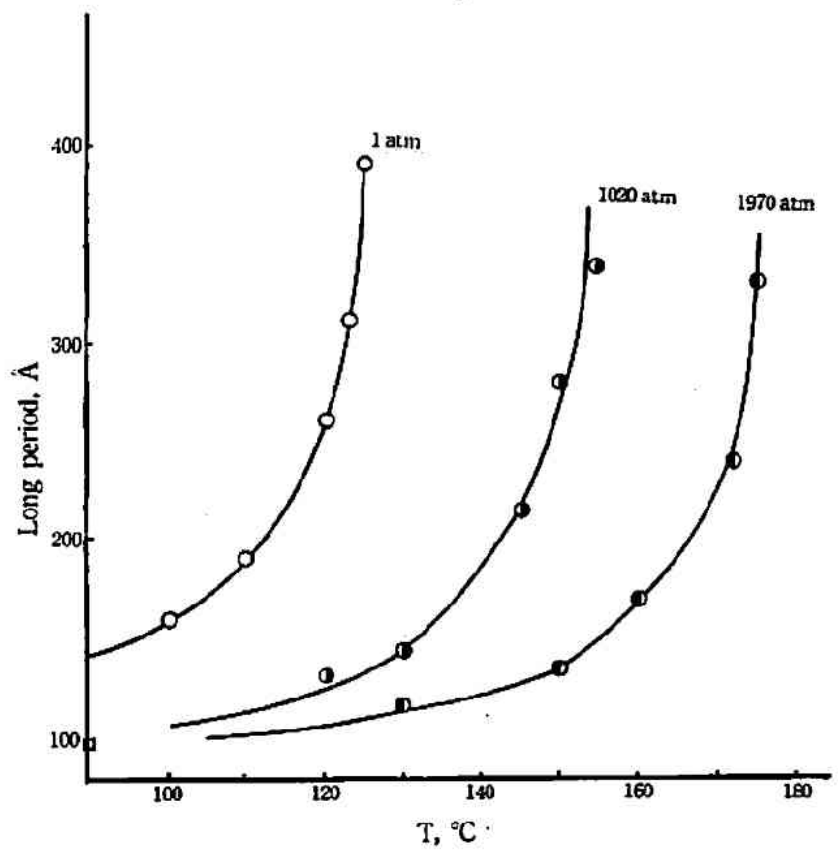

Fig. 3 Thickness of single crystal lamela as a function of annealing temperature at various pressures

and that of the original lozenge shape printed on the collodion membrane shows that the former thickened about 2 times. Small angle $x$-ray diffraction for the sample annealed under the same

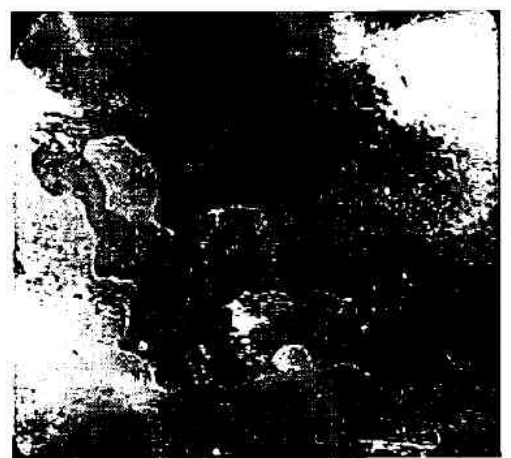

Photo. I Electron micrograph of a single crystal of Marlex 50 heated at $120^{\circ} \mathrm{C}$ for $2 \mathrm{hrs}$ under atmospheric pressure

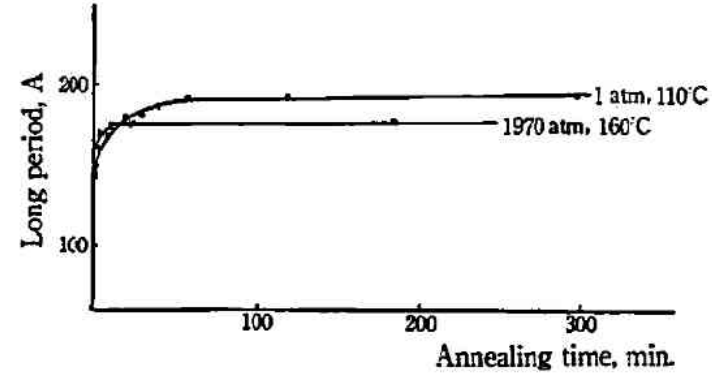

Fig. 4 Increase in thickness of Marlex 50 single crystals during annealing 
condition showed that the thickness of the crystallites was about $220 \mathrm{~A}$. The morphological features of the single crystal annealed under high pressure would be the same as those at the atmospheric pressure. The thickness of the single crystals of polyethylene treated under va-ious pressures is shown in Fig. 3. It is shown that the annealing temperature $T\left({ }^{\circ} \mathrm{C}\right)$ and pressure $P(a t m)$ have a pronounced influence on the thickness $L(A)$. The values of $L$ increase with $T$ slowly in the lower temperature range and steeply in the higher temperature range near the melting points for each pressure. The annealing time does not affect the thickening process so much when it is above about 2 hrs as shown in Fig. 4.

(2) Electron microscope observation

The fracture surface of the sample crystallized at $160^{\circ} \mathrm{C}$ under 1100 atm is shown in Photo. 2 . In the large portion of the photograph, the band structure of fairly uniform thickness can be observed. This structure represents the lamellae which are seen edge-on. The polymer molecules are folded in order to fit into the band structure and the molecular chain axes are essentially perpendicular to the lamellar thickness. The striations of the order of $300 \mathrm{~A}$ in width normal to the band structure would not be the molecule itself, but might be attributed to the molecular chain bandles. They are, however, beneath the resolving power of the replicas.

The thickness of lamellae is in the order of 300 to $1000 \mathrm{~A}$. The whole length of the molecular chain of Marlex 50 is about $5000 \mathrm{~A}$, and so the molecules are extremely extended as long as $1000 \mathrm{~A}$ but not fully extended and folded in these bands. It is also interest to notice that the fold length of $1000 \mathrm{~A}$ is consistent with the value expected from the lamellar thickness of single crystals annealed under high pressure. The point $\left(\mathrm{L}=1000 \mathrm{~A}\right.$ and $\left.\mathrm{T}=160^{\circ} \mathrm{C}\right)$ is almost on the extrapolated straight line of $\mathrm{L}$ ขs. $1 /\left(\mathrm{T}_{\mathrm{m}}-\mathrm{T}\right)$ plot for $\mathrm{P}=1100 \mathrm{~atm}$ as shown in Fig. 5.

The dark fibrous matters of about $300 \mathrm{~A}$ in width which are seen in the whole range and predominantly in the lower righthand side of Photo. 2 may be the fibers in which the molecules are pulled out from the folded-chain lamellae. Pulling-out was carried out so rapidly that the energy produced was dissipated as heat. This heating causes the fbrils to melt at their ends and ball up in the manner seen in Photo. 2.

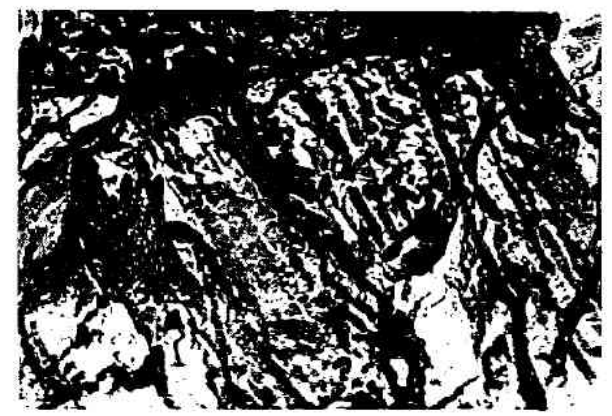

Photo. 2 Fracture surface of poly. ethylene (unfractionated Marlex 50) crystallized at $160^{\circ} \mathrm{C}$ under $1,125 \mathrm{~atm}$

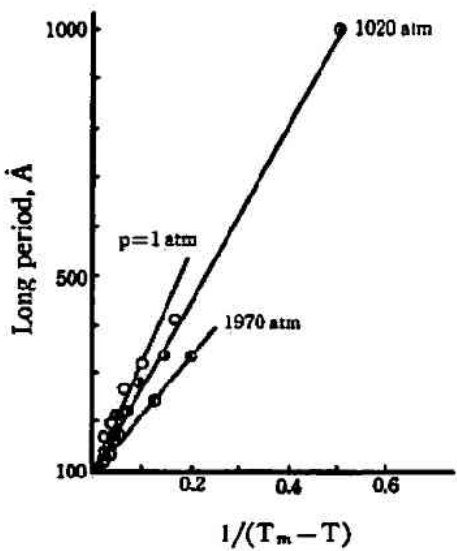

Fig. 5 Plot of $L$ against $1 /\left(\mathrm{T}_{m}-\mathrm{T}\right)$ for Marlex 50 crystallized at various pressures 


\section{Consideration}

According to the theory of Lauritzen-Hoffman,4) L, the thickness of the lamellae of the single crystals crystallized from melt at the temperature $T$, is given by the following equation.

$$
\mathrm{L}=\frac{k \mathrm{~T}}{d_{s} \sigma_{\varepsilon}}+\frac{2 \sigma_{r}}{\Delta f_{u}}
$$

Here, $d_{0}$ is the diameter of the chain molecule, $\sigma_{s}$ and $\sigma_{c}$ the surface free energies per unit area of the lateral and the end surfaces of lamellae respectively, and $\Delta f_{w}$ the free energy of crystallization which is represented by

$$
\Delta f_{\mathrm{u}}=\Delta h\left(\mathrm{~T}_{m}-\mathrm{T}\right) / \mathrm{T}
$$

where $\Delta h$ is the heat of fusion per unit volume of the polymer. Thus eq. (1) is deduced to the simple equation

$$
\mathrm{L}=\mathrm{L}_{n}+\frac{\mathrm{A}}{\mathrm{T}_{m}-\mathrm{T}}
$$

with $\mathrm{A}=2 k \mathrm{~T} / d_{0 \sigma}$, and $\mathrm{L}_{0}=k \mathrm{~T} / d_{\circ} \sigma_{s}$,

in which $L_{0}$ is the value of $L$ when $T$ is comparably close to $T_{m}$. The data of the lamellar thickness $\mathrm{L}$ in Fig. 3 are replotted against $1 /\left(\mathrm{T}_{n t}-\mathrm{T}\right)$ in Fig. 5 . We have good straight lines for each pressure in these temperature range as eq. (3) requires. The values of $T_{m}$ are decided by the asymptotes of each curve in Fig. 3. This result implies that the crystallization process under elevated pressure seems to be ruled by the mechanism of Lauritzen-Hoffman as in the case of the atmospheric pressure. From the intercept and the inclination of the straight line, the values of $L_{o}$ and $A$, and accord-

\begin{tabular}{|c|c|c|c|c|}
\hline$P($ atm $)$ & $L_{o}(A)^{*}$ & A(A/deg. $)$ & $\mathrm{T}_{m\left({ }^{\circ}\right.}(\mathrm{C})$ & $\sigma_{0}\left(\mathrm{erg} / \mathrm{cm}^{2}\right)$ \\
\hline 1 & 96 & 2200 & 140 & 77 \\
\hline 1020 & 90 & 1850 & 161 & 62 \\
\hline 1970 & 83 & 1350 & 185 & 43 \\
\hline
\end{tabular}
ingly $\sigma$, were calcuated, which are listed in Table 1.

Table 1

* Calculated values at $\mathrm{T}_{\mathrm{m}}-\mathrm{T}=50$

The melting point $\mathrm{T}_{m}$ should be elevated with increasing pressure $\mathrm{P}$ and may be predicted from the Clapeyron equation;

$$
\frac{d \mathrm{P}}{d \mathrm{~T}_{m}}=\frac{\Delta H}{\mathrm{~T}_{m} \Delta V} \text {, }
$$

where $\Delta H(\mathrm{cal} / \mathrm{g})$ denotes the latent heat of fusion, $\mathrm{T}\left({ }^{\circ} \mathrm{K}\right)$ the melting point. and $\Delta V(\mathrm{cc} / \mathrm{g})$ the volume change of fusion. At least in the pressure iange of the present experiment, $\Delta H$ and $\Delta V$ may be assumed to be constant, and eq. (4) can he approximated by

$$
\mathrm{P}=\frac{\Delta H}{\Delta V} \ln \frac{\mathrm{T}_{m}}{\mathrm{~T}_{m}^{1}} \fallingdotseq \frac{\Delta H}{\mathrm{~T}_{m} \Delta V}\left(\mathrm{~T}_{m}-\mathrm{T}^{\mathrm{l}}\right)
$$

4) J. D. Hoffman and J. I. Lauritzen, Jr., J. Res. N. B. S., 65A, 297 (1961) 
where $\mathrm{T}_{\mathrm{m}}{ }^{1}$ is the melting temperature at the atmospheric pressure. Now put $\Delta H=68.0 \mathrm{cal} / \mathrm{g}$, $\Delta V=0.16 \mathrm{cc} / \mathrm{g}$ and $\mathrm{T}^{\mathrm{t}}{ }_{\mathrm{w}}=410^{\circ} \mathrm{K}$, then we heve

$$
\mathrm{P}(\mathrm{atm})=42.5\left(\mathrm{~T}_{m}-410\right) \text {. }
$$

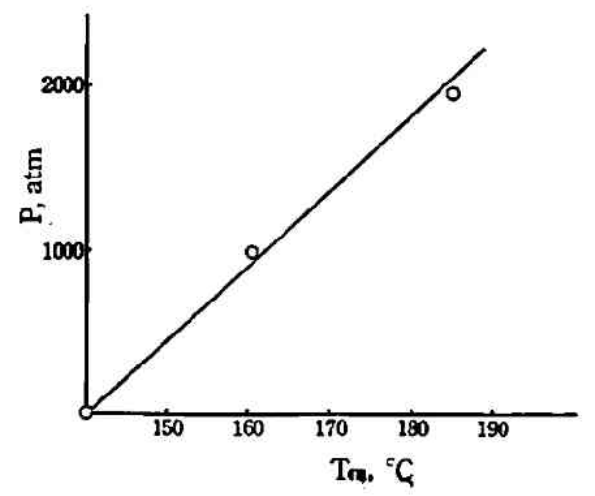

Fig. 6 Effect of pressure $(\mathrm{P})$ on the melting point $\left(\mathrm{T}_{\mathrm{m}}\right)$ of polyethylene; $(\mathrm{O})$ experimental points. The solid line shows eq. (6).

The values of $\mathrm{P}$ and $\mathrm{T}_{m}$ listed in Table 1 are in accord well with this equation as shown in Fig. 6, and therefore they are considered to be adequate as the melting temperatures for the given pressures.

The values of $\sigma_{s}$ calculated from eq. (3) are less than $10 \mathrm{erg} / \mathrm{cm}^{2}$ which are almost the same order as the surface free energy of liquid paraffins, but the values of $\sigma_{e}$ calculated are much larger. The value of $\sigma_{0}$ can be estimated from the molecular conformation of the chain folding which is considered to contain four gauche configurations in it. The excess energy for a fold is $700 \times 4$ cal., where $700 \mathrm{cal}$. is the energy difference between the trans and the gauche forms. Since the surface area for a fold is equal to $36.5 \times 10^{-16} \mathrm{~cm}^{2}$, the excess surface energy per unit area of the end surface is $60 \mathrm{erg} / \mathrm{cm}^{2}$, and the value of $\sigma$, becomes $60+10=70 \mathrm{erg} / \mathrm{cm}^{2}$ which agrees well with the values listed in Table 1.

Eq. (3) shows that $A$ is proportional to $T_{m}$ and so it is expected to decrease with pressure, but the reverse is true as seen in Table 1 . This means that the surface free energy of the end surface of the single crystal $\sigma_{t:}$ would decrease with increasing pressure as is represented in Table 1 . It is doubtful whether any quantitative treatment of this fact is possible as yet, but it is reasonable to assume that the energy difference between the gauche and trans configurations becomes less under high pressure.

Laboratory of Physical Chemisiry

Department of Chemistry

Faculty of Science

Kyoto University

Kyoto, Japan 